\section{Análisis de la composición química de litiasis urinaria de 649 pacientes mediante espectroscopía infrarroja, primera experiencia nacional}

\author{
RODRIGO SÁNCHEZ ${ }^{1}$, PABLO NAVARRO²,3,a,, MARÍA PÍA TRONCOSO ${ }^{4}$, \\ CAMILA LÓPEZ ${ }^{4}$, JOSÉ ANTONIO SALVADÓ 5
}

\section{Chemical composition analysis of 649 urinary stones}

Background: Chemical composition analysis of urinary stones is a fundamental part of the metabolic workup of urolithiasis. Aim: To report the chemical composition of urinary stones using infrared spectroscopy. Material and Methods: The chemical composition of rinary stones recovered from 649 patients aged 1 to 97 years (68\% males), were analyzed using a Perkin Elmer FTIR Spectrometer, Spectrum Two. Results: Calcium oxalate monohydrate was the most common composition found in $45 \%$ of cases, followed by mixed composition, which included three ammonium phosphate stones in $29 \%$ of cases. Pure uric acid composition was found in $16 \%$ of stones. Three cystine stones were detected. Conclusions: These findings do not differ from those found in developed countries.

(Rev Med Chile 2021; 149: 1129-1133)

Key words: Calcium Oxalate; Spectrophotometry; Urolithiasis.
'Universidad Finis Terrae,

Santiago, Chile. Servicio de Urología Clínica Santa María. Santiago, Chile.

${ }^{2}$ Centro de Investigación en

Ciencias Odontológicas, Facultad de Odontología, Universidad de La Frontera. Temuco, Chile. ${ }^{3}$ Universidad Autónoma de Chile. Temuco, Chile.

${ }^{4} U$ niversidad Finis Terrae.

Santiago, Chile.

${ }^{5}$ Servicio Urología Clínica Santa María. Centro de Estudio y

Manejo de la Litiasis Urinaria,

Clínica Santa María, Santiago, Chile. Universidad Finis Terrae.

Santiago, Chile.

angeniero Comercial.

bPhD en Estadística.

Los autores declaran no tener conflictos de interés. Trabajo no recibió

financiamiento.

Recibido el 21 septiembre de 2020, aceptado el 19 de mayo de 2021.

Correspondencia a: Rodrigo Sánchez Sepúlveda Evaristo Lillo 29, Departamento 67. Las Condes. Santiago Rsanchez@clinicasantamaria.cl
L a nefrolitiasis es una de las condiciones urológicas más comunes. La prevalencia durante la vida se estima en $1 \%$ a $15 \%$, dependiendo de la edad, genero, raza y ubicación geográfica ${ }^{1}$. El riesgo de sufrir durante la vida un episodio sintomático secundario a litiasis urinaria ha ido en aumento en las últimas décadas, afectando aproximadamente a 10\% de la población en países occidentales ${ }^{2}$. En relación con lo anterior, reportes del hemisferio norte han señalado que este cuadro produce una carga económica mayor a los 5 billones de dólares en gastos directos e indirectos ${ }^{3}$, estimando incluso que induce un gasto promedio de $\$ 3.500$ por persona al año ${ }^{4}$.

El componente más frecuente de la litiasis urinaria es el calcio, el cual se encuentra presente en alrededor de $75 \%$ de los cálculos, aproximadamente $60 \%$ están compuestas por oxalato de calcio, composiciones mixtas de calcio e hi- droxiapatita representan 20\% y ácido úrico 10\%, estruvita se encuentra cercano a $10 \%$, brushita $2 \%$ y cistina $1 \%{ }^{4}$.

El análisis de la litiasis urinaria es considerado parte fundamental en la evaluación para la prevención de recurrencia, tal es así que las actuales guías clínicas europeas estiman que el punto de inicio para definir riesgo de recurrencia en un paciente litiásico es el análisis químico del cálculo por algún método validado (espectroscopía o difracción de rayos $\mathrm{X})^{5}$. Por otra parte, ciertas composiciones químicas de una litiasis pueden influenciar negativamente el éxito de un tratamiento extracorpóreo por ondas de choque ${ }^{6}$, por lo que el conocer la composición en pacientes litiásicos recurrentes permitiría orientar a tratamientos endoscópicos laser, cuyas tasas de éxito no se ven influenciadas por la naturaleza química de la piedra.

El objetivo del presente estudio es reportar la 
composición química de los cálculos urinarios obtenidos a partir de la mayor base de datos a nivel nacional del análisis de litiasis urinaria mediante espectroscopía infrarroja en un centro de atención privado de salud.

\section{Materiales y Métodos}

En el año 2017, nuestro centro llevo a cabo la incorporación del Espectómetro FTIR Perkin Elmer, siendo este el primero a nivel nacional. Se realizó un estudio descriptivo, retrospectivo de un total de 649 pacientes sometidos a análisis de litiasis como parte del estudio metabólico, los cuales fueron realizados con el uso de Espectrómetro FTIR Perkin Elmen, Spectrum two entre junio del 2017 y junio del 2019. Los procedimientos respetaron las normas éticas concordantes con la Declaración de Helsinki, fueron revisados y aprobados por el Comité de Ética de Clínica Santa María en el contexto de análisis de litiasis mediante espectroscopía infrarroja y del estudio metabólico en pacientes de dicho centro asistencial. Se solicitó dispensa de uso de consentimiento informado por el carácter retrospectivo del análisis, consiguiendo dicha autorización. Las muestras obtenidas para el estudio fueron aquellas recuperadas por expulsión espontánea del cálculo por parte del paciente, o en el contexto de intervención quirúrgica (Litotripsia extracorpórea, Nefrolitectomía percutánea y ureterolitotomía endoscópica). Se evaluaron los resultados según género, tipo de cálculo (Oxalato de Calcio monohidrato [COM], Oxalato de Calcio dihidratado [COD], Ácido úrico, Cistina, Fosfato de Calcio, Mixtos [incluye más de uno de los compuestos anteriores y los cálculos con componente de fosfato de amonio] y se dividieron a los pacientes según grupos de edad y tipo de cálculo.

\section{Análisis estadístico}

La recolección de los datos se registró en una planilla Microsoft Office Excel, se realizó un análisis descriptivo de los datos para los que se determinó tablas de distribución de frecuencias para las variables en estudio. Se realizó la prueba no paramétrica Chi-Cuadrado para variables cualitativas. Para el análisis de los datos se usó el programa estadístico IBM SPSS Statistics (versión $20,0)$. Un valor de $\mathrm{p}<0,05$ fue elegido como umbral para significancia.

\section{Resultados}

Un total de 649 muestras de pacientes fueron analizados (un análisis por paciente). La gran mayoría de las muestras provienen de pacientes de sexo masculino, a una razón 2,16:1. El promedio de edad fue de 45 años, con un rango entre 1-97 años. Sólo 3,1\% de los individuos tenían menos de 20 años, siendo el grupo etario más representado en esta muestra aquellos de entre 41-60 años, representando $46,8 \%$ del total.

La composición más frecuente en este estudio fue el Oxalato de Calcio monohidrato (COM, $\mathrm{n}=294$ ), seguido por la composición mixta ( $\mathrm{n}=186$, dentro de estos destacan los 11 cálculos mixtos con componente predominante de fosfato de calcio o brushita, establecido de forma arbitraria como más de $50 \%$ de dicho componente, los 3 de componente predominante de fosfocarbonato de calcio o carbapatita, y también se incluyen aquí 3 cálculos con componente de fosfato de amonio) y ácido úrico $(\mathrm{n}=103)$, fosfato de calcio dihidratado $(\mathrm{COD}, \mathrm{n}=33)$, fosfato de calcio puro $(\mathrm{n}=30)$ $\mathrm{y}$ cistina $(\mathrm{n}=3)$.

En la Tabla 1 se describen los pacientes, sus rangos etarios y los componentes estudiados.

Al comparar los tipos de cálculos según género (Figura 1), el género masculino aportó un mayor número muestras en esta serie con una marcada relación al componente de ácido úrico y cistina en menor grado, al igual que con el componente

Tabla 1. Descripción de los 649 pacientes estudiados y la composición de los cálculos evaluados mediante espectroscopía infraroja

\begin{tabular}{|llrr|}
\hline Sexo & Femenino & 206 & $(31,7 \%)$ \\
& Masculino & 443 & $(68,3 \%)$ \\
Tipo de cálculo & Ácido úrico & 103 & $(15,9 \%)$ \\
& COD & 33 & $(5,1 \%)$ \\
& COM & 294 & $(45,4 \%)$ \\
& Cistina/colcistina & 3 & $(0,5 \%)$ \\
& Fosfato de calcio & 30 & $(4,6 \%)$ \\
& Mezcla & 186 & $(28,7 \%)$ \\
Edad & & 45 & $(35-56)$ \\
Grupo etario & $\leq 20$ & 20 & $(3,1 \%)$ \\
& $21-40$ & 224 & $(34,6 \%)$ \\
& $41-60$ & 303 & $(46,8 \%)$ \\
& $\geq 61$ & 100 & $(15,5 \%)$ \\
\hline
\end{tabular}

Oxalato de Calcio Monohidrato (COM), Oxalato de Calcio Dihidratado (COD). 
de oxalato de calcio monohidrato, identificándose una diferencia estadísticamente significativa al realizar la comparación con el género femenino, con un $\mathrm{p}=0,001$.
Al realizar el análisis entre el tipo de cálculo y el grupo etario de los pacientes, destaca que el mayor número de muestras proviene de individuos entre 40 y 60 años (Figura 2), destacando

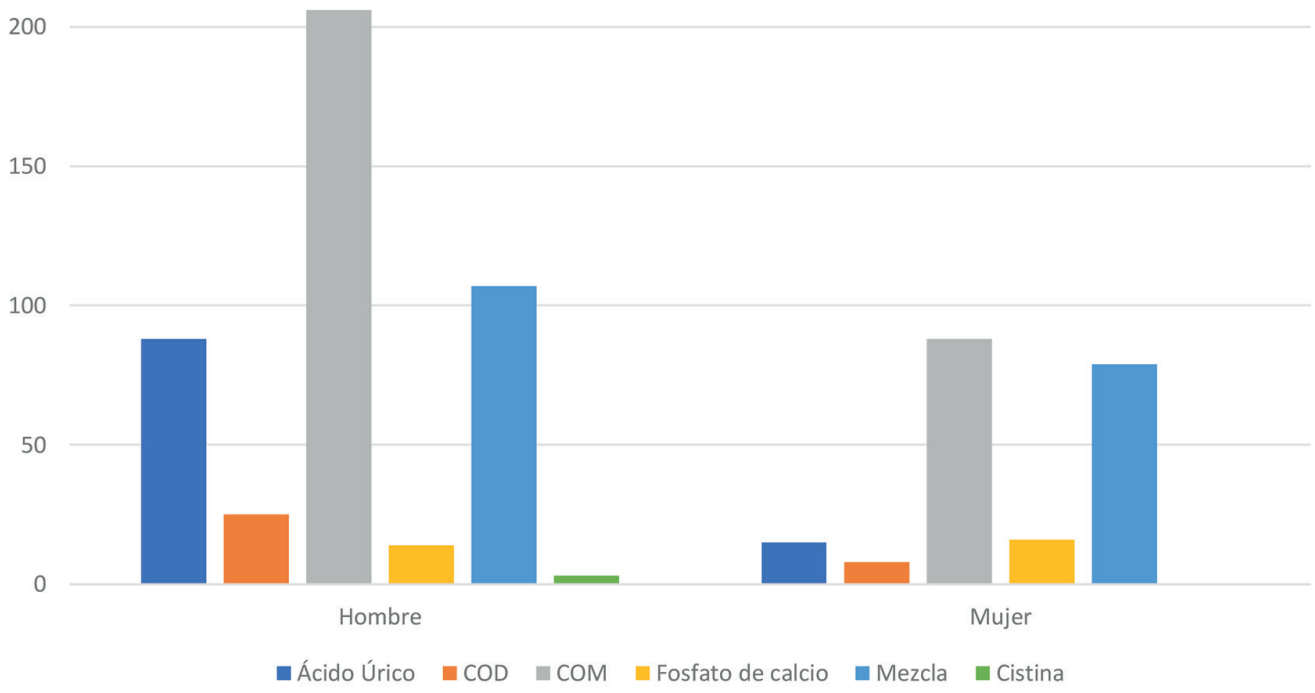

Figura 1. Distribución de litiasis urinaria según sexo y tipo. Oxalato de Calcio Monohidrato (COM), Oxalato de Calcio Dihidratado (COD).

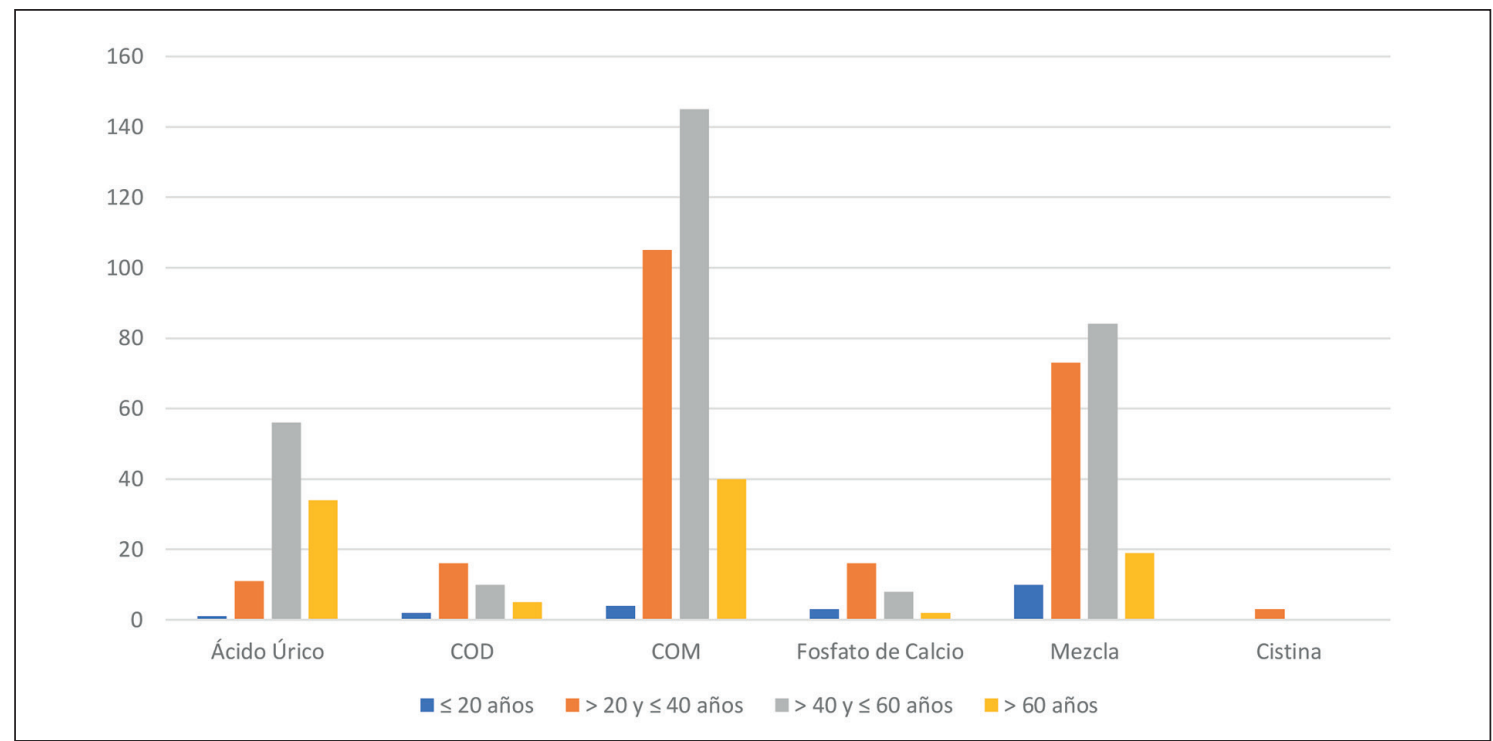

Figura 2. Distribución de litiasis urinaria según tipo y edad. Oxalato de Calcio Monohidrato (COM), Oxalato de Calcio Dihidratado (COD). 
sobre todo los cálculos de COM, mixtos y ácido úrico. Por otra parte los 3 cálculos de cistina en esta serie corresponden a un paciente de 21 años y dos de 23 años. De igual forma, en nuestro estudio hubo solo 3 cálculos de etiología infecciosa (fosfato de amonio), representando $0,46 \%$ de la muestra, muy lejos de $10 \%$ habitualmente descrito en la literatura. Es por ello que, en esta serie, existen diferencias estadísticamente significativas entre el tipo de litiasis y el rango etario, con un $\mathrm{p}=0,002$.

\section{Discusión}

El análisis químico de la litiasis es importante por varias razones. La principal es que entrega directrices sobre las causas subyacentes sobre la aparición de cálculos urinarios, lo que permitiría prevenir recurrencias, lográndose esto último con un adecuado estudio metabólico ${ }^{7}$ Existen diferentes métodos validados para realizar este análisis. Estos se dividen habitualmente en métodos químicos y físicos. Dentro de los métodos químicos, se encuentra el análisis húmedo de la litiasis, siendo una de las técnicas más utilizadas. El gran problema de este método, al igual que los otros métodos químicos, este sólo puede identificar la presencia de iones y radicales de forma individual, sin poder lograr la identificación precisa de los componentes ni elementos mixtos en un mismo cálculo $^{8}$. Es por lo anteriormente mencionado, que los métodos químicos han pasado al desuso, siendo actualmente los más utilizados los métodos físicos. La ventaja que ofrecen estos métodos, dentro de ellos la espectroscopía infrarroja, es que permite identificarcada uno de los componentes y sus proporciones a través de una evaluación semi cuantitativa ${ }^{2}$.

Además, estos métodos permiten identificar litiasis no cálcicas, tales como cistina, xantina, ácido úrico, estruvita, litiasis secundarias a fármacos, así como tambiénde las litiasis de oxalato de calcio y fosfato de calcio ${ }^{2}$.

La espectroscopía infrarroja, introducida en 1955, utiliza la radiación infrarroja causando la vibración de los átomos, provocando absorción de la energía producida para generar, en última instancia, bandas de absorción en el espectro de la luz infrarroja ${ }^{9}$. Este método es el más utilizado actualmente, siendo nuestro centro el primero en incorporarlo a nivel nacional para realizar este tipo de estudios.

La urolitiasis es una enfermedad altamente prevalente y costosa para los servicios de Salud.

En el año 2012 Scales $^{11}$ reportó una prevalencia en Estados Unidos de 8,8\%, al analizar por género, la prevalencia en los hombres resultó mayor que en las mujeres ( $10,6 \%$ vs $7,1 \%)$, Si bien en nuestro país no existen estudios de prevalencia de litiasis urinaria, nuestro análisis es similar a la distribución antes mencionada, con un predominio de muestras aportadas por pacientes de género masculino, alcanzando a $68 \%$ de la muestra. En relación con los costos y el aumento en la prevalencia de diabetes y obesidad, se cree que para el 2030, el gasto asociado al manejo de la enfermedad litiásica aumentará en US $\$ 1,24$ billones anuales ${ }^{12}$, de ahí la importancia del estudio metabólico y análisis espectroscópico, como complemento del estudio global de estos pacientes, en la evaluación de esta enfermedad.

Un análisis de 4.399 litiasis mostró que el principal componente de los cálculos urinarios correspondía en primer lugar al COM (presente en $93 \%$ de las litiasis), seguido de COD (en 57\%) y ácido úrico en $12 \%$ de los cálculos ${ }^{11}$. Este estudio concluye que la composición de ácido úrico aumenta proporcionalmente respecto al grupo etario analizado, hallazgo ya descrito por Leske ${ }^{13}$ y Usman ${ }^{14}$; y la incidencia de COD disminuye con el paso de los añosy la de COM se mantiene relativamente alta en todos los grupos de edades, hallazgos semejantes a la distribución de COM

Cabe destacar que, en nuestra serie, hay un alza de litiasis de ácido úrico en el grupo entre los 40 y 60 años comparado con los pacientes entre 0 y 40 años, sin producirse un alza marcada en mayores de 60 años, aunque, de todas maneras, las litiasis de ácido úrico representan el segundo componente más frecuente $(n=40)$ en ese rango etario.

Otro análisis de 1.254 cálculos mediante espectroscopía infraroja detectó $53,7 \%$ de litiasis de oxalato de calcio monohidrato, los cuales estaban presentes mayoritariamente en hombres. El resto se distribuyó en 17,6\% de litiasis correspondientes a oxalato de calcio dihidratadoy $8,9 \%$ de cálculos de ácido úrico (con un mayor número de casos, aunque no significativo, en el género masculino), aproximadamente $1 \%$ de litiasis de cistina y brushita en $2,5 \%{ }^{15}$. Un hallazgo un poco diferente se evidencia en nuestro estudio, el que demostró una 
diferencia estadísticamente significativa, en favor de los hombres, tanto en las litiasis de oxalato de calcio monohidrato como las de ácido úrico y cistina y un porcentaje mucho menor de cálculos de brushita, con un total de 41 litiasis de fosfato de calcio (incluidos los de fosfato de calcio puro y los de composición mixta cuyo elemento principal era brushita), representando 6,31\% del total de litiasis.

Nuestra serie inicial, con pocos casos aún, se comporta de manera similar a estudios de países desarrollados, en termino de predominancia de ciertas componentes por sobre otros e indirectamente en frecuencia de análisis al comparar género y edades. Esto estaría probablemente relacionado con nuestro comportamiento epidemiológico, hábitos alimenticios, deportivos, y la tendencia al alza de enfermedades crónicas asociadas a litiasis, las que han ido adquiriendo un comportamiento similar a la de países desarrollados. Un sesgo de nuestro estudio podría estar asociado a un potencial mayor nivel socio económico de los pacientes, ya que se trata de un servicio privado de salud. Asimismo, debemos considerar otras limitaciones de nuestro reporte, puesto que se trata de un análisis retrospectivo y descriptivo, realizado en un solo centro, lo que no necesariamente es representativo del comportamiento similar a nivel nacional

No obstante, este estudio permite resaltar la importancia del análisis de los componentes químicos de la litiasis mediante espectroscopía infrarroja, para determinar la patogénesis y etiología de un cálculo determinado y poder así ofrecer directrices de tratamiento y manejo a los pacientes según su riesgo individual ${ }^{16}$.

A modo de conclusión, este estudio, primero en su tipo a nivel nacional y con la serie más grande de pacientes documentada hasta la fecha, demuestra que el principal componente de litiasis es el oxalato de calcio monohidrato, siendo el grupo predominante hombres adultos.

\section{Referencias}

1. Pearle M, Antonelli J, Lotan Y. Urinary Lithiasis: Etiology, Epidemiology, and Pathogenesis. En:Partin A, Peters C, Kavoussi, Domochowski R, Editores, Campbell-Walsh-Wein, Urology. Filadelfia, Estados Unidos; Editorial
Elsevier.; 2020. p 2005-44.

2. Cloutier J, Villa L, Traxer O, Daudon M. Kidney Stone Analysis: "Give me your Stone, I well tell you who you are!” World J Urol 2015; 33(2): 157-69.

3. Boydston K, Terry R, Winship B, Davis L, Yttri S, Carlos $\mathrm{E}$, et al. The Impact of Alternative Alkalinizing Agents on 24 - Hour Urine Parameters. Urology 2020; 142: 55 9.

4. Saigal CS, Joyce G, Timilsina AR. Urologic Diseases in America Project. Direct and indirect costs of nephrolithiasis in an employed population: opportunity for disease management? Kidney Int 2005; 68(4): 1808-14.

5. Türk C, Neisius A, Petrik A, Seitz C, Skolarikos A, Thomas K, et al. EAU Guidelines on Urolithiasis. European Association of Urology 2020; 12-3.

6. Madaan S, Joyce AD. Limitations of extracorporeal shock wave lithotripsy. Curr Opin Urol 2007; 17: 19013 .

7. Singh I. Renal geology (quantitative renal Stone analysis) by "Fournier transform infrared spectroscopy". Int Urol Nephrol 2008;40: 595-602.

8. Kasidas GP, Samuell CT, Weir TB. Renal Stone analysis: why and how? Ann Clin Biochem 2004; 41: 91-7.

9. Basiri A, Taheri M, Taheri F. What is the State of the Stone Analysis Techniques in Urolithiasis? Urol J 2012; 9 (2): 445-54.

10. Scales CD, Smith AC, Halney JM, Sigal CS. Urologic Diseases in America Project. Prevalence of Kidney Stones in the United States. Eur Urol. 2012; 62: 160-5.

11. Ziemba J, Matlaga R. Epidemiology and economics of nephrolithiasis. Investig Clin Urol. 2017; 58: 299-306.

12. Grant C, Guzman G, Stainback RP, Amdur RL, Mufarrij P. Variation in Kidney Stone Composition within the United States. J Endourol. 2018; 32: 973-7.

13. Lieske JC, Rule AD, Krambeck AE, Williams JC, Bergstralh EJ, Mehta RA, et al. Stone composition as a function of age and sex. Clin J Am Soc Nephrol. 2014; 9: 2141-6.

14. Usman KD, Golans S, Abdin T, Livne PM, Pode D, Duvdevani M, et al. Urinary Stone Composition in Israel: Current Status and Variation with Age and Sex-A Bicenter Study. J Endourol. 2013; 27: 1539-42.

15. Kravdal G, Helgo D, Moe M. Kidney Stone Compositions and Frequencies in a Norwegian Population. Scandinavian Journal of Urology. 2019; 53: 139-44.

16. Mandel N, Mandel I, Kolbach-Mandel A. Accurate Stone Analysis: the impact on disease diagnosis and treatment. Urolithiasis. 2017; 45: 3-9. 